\title{
MULTI-CRITERIA MODELS IN AUTONOMOUS DECISION MAKING SYSTEMS
}

\author{
Karel Mls* \\ Faculty of Informatics and Management \\ University of Hradec Kralove \\ Hradec Kralove, Czech Republic \\ E-mail: karel.mls@uhk.cz \\ Martin Gavalec \\ Faculty of Informatics and Management \\ University of Hradec Kralove \\ Hradec Kralove, Czech Republic \\ E-mail: martin.gavalec@uhk.cz
}

\begin{abstract}
Decision making processes, based on various multi-criteria models are considered. The main differences between AHP (Analytic Hierarchy Process), ANP (Analytic Network Process) and CHP (Cognitive Hierarchy process) methods will be discussed. The possible use of referred methods for decision support in autonomous systems will be proposed and strong and week points of the methods will be experimentally demonstrated on on-line decisional problem.
\end{abstract}

Keywords: AHP, ANP, CHP, autonomous system, on-line decision making

\section{Introduction}

Computerized decision making support history is commonly considered to begin in the 1960s, when Michael S. Scott Morton has published his dissertation (Scott Morton, 1967). While the idea of "mancomputer symbiosis", stimulated by fast evolution of personal computers, has thrilled scientists and academicians, managers were more skeptical in accepting the novelty. Managers' caution might been caused by the experience with preceding so called Management Information Systems and other suggested computer-based tools and their problematic benefits for the practice. Today, the situation is changing accelerating development and availability of the hardware base subsequently enable to create practically efficient applications - web-based and intelligent Decision Support Systems (DSS), actually incorporated into the Business Intelligence framework - but skepticism of many potential users still remains.

Together with the DSS applications development, academic research of the decision theory and decision methods were widely conducted (Alter, 1975), (Sprague \& Carlson, 1982), (Drucker et al. 2001), (Power, 2002). Many other researchers have consequently focused their attention to the new and promising topic.

While tactical decisions, distinguished by semi structured problems, can be successfully managed by aforesaid DSS, there are still open problems in strategic decision making support (Mullins, Walker \& Boyd, 2006). Strategic decisions are hard to describe in formal way, as there are individual and subjective characteristics, which are difficult or impossible to formalize, and therefore some authors consider strategic decision making more as an art than as the science (Mintzberg \& Quinn, 1988), (David, 1989),

\footnotetext{
* The support of Czech Science Foundation \# 402/09/0405 is kindly acknowledged
} 
(Hussey, 2001). Conditions for decision making are becoming more and more complex at the same time; there is unlimited quantity of data available, but less time to process them. The use of modern information technologies can help to overcome some of these difficulties (Torra, Nakurawa \& Yoshida, 2007), but the importance of the manager's competency is still unexceptionable.

Although there are many methods, how to train decision-making skills (Fernández-Aráoz, 2007), strategic decisions are distinguished by unique, case specific conditions. To solve such tasks in situations, when there is no competent decision maker (space missions) or when the decision is emotionally difficult for humans (military or rescue operations), intelligent and autonomous decision making systems were suggested (Pollock, 2006).

Autonomous systems are progressively becoming integral part of many technical and economical solutions. While their technical appearance, represented mainly by autonomous humanoid robots, attracts general attention (Holland, 2003), (Bekey, 2005), (Thrun, 2005), there are many other possible daily applications of autonomous systems in control and business. Further research and development in the considered field is under strong focus now.

\section{Decision support technologies}

From the very beginning of the DSS utilization, the crucial role of the user - decision maker - in adopting the final decision was declared. Therefore, DSS designs, from classical to intelligent ones, with respect to this paradigm, were based on the conceptually similar scheme (Figure 1). The user has access to all available data by means of the Data Subsystem, where not only query, but also analytical tools are present. On the other hand, Model Subsystem is supporting more specific analyses, predictions and simulations by domain specific models, necessitated by the user. Such systems were built pursuant to the theory of rational decision making and the user is therefore forced to decide rationally, too.

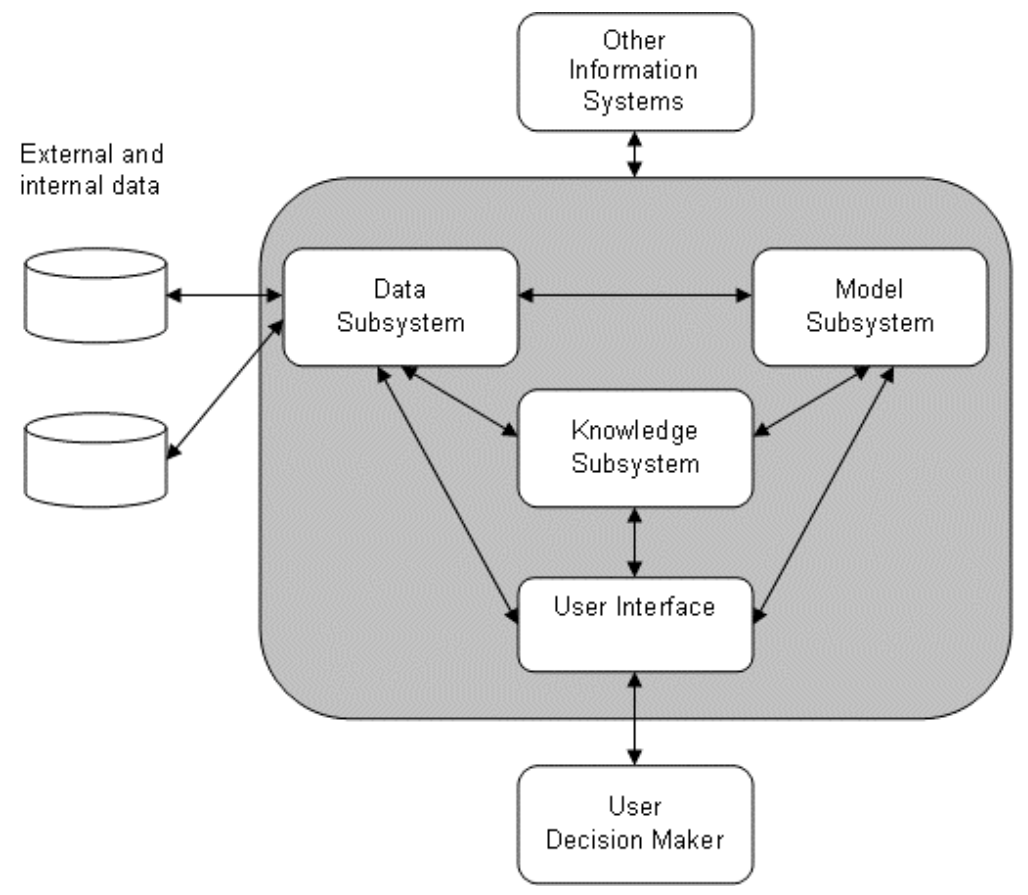

Figure 1. Conceptual model of a Decision Support System (Turban, 1995). 
First attempts at usage of autonomous decision systems in relatively simple or well structured environments, represented for example by logistic processes (Jedermann et al, 2007), supply chain simulations (Keller, Duguay \& Precup, 2004), computer networks, health care networks and, undoubtedly, by military operations may serve as a baseline for future progress in the presented field.

\subsection{Analytic Hierarchy Process}

Analytic Hierarchy Process (AHP) is a method developed for creating structured models of multi-criteria decision problems. The method helps to find an alternative which suits best the given needs of the deciding person. Analyzing the set of possible alternatives, the AHP method finds the one with the best rating, based on the structure of the problem and given preferences. Saaty formulated the principles of AHP in late 1970s (Saaty, 1980), and the method has been broadly studied and applied in many cases since the time. The method combines mathematical and psychological aspects, starting with defining the structure of the problem, then quantifying the relative preferences, computing the priorities and finally computing the evaluation of all considered alternatives. First of all, the multi-criteria decision problem is converted into a hierarchy of sub-problems and every of the sub-problems is then independently analyzed. The criteria of the sub-problems in the hierarchy may have very heterogeneous nature, they may be precisely or vaguely defined, with crisp or fuzzy parameters, formal or intuitive, etc. The relative preferences of heterogeneous criteria are then quantified by human decision-maker using his/her ability of comparing various aspects of the problem. The decision maker systematically compares the criteria in pairs and quantifies the relative importance either by available data or by intuitive judgment. The relative preferences found in pairs are then used to compute weights (priorities) for every part of the hierarchy model. The evaluation computed for all decision alternatives then shows their relative strength from the point of view of the entire problem. It is the advantage of AHP that even considerably diverse criteria can be used in the model, and that not only exact data but also human judgments can be applied to describe various aspects of the problem.

\subsection{Analytic Network Process}

The Analytic Network Process (ANP) is seen as a generalization of the AHP. Interaction and dependence among higher-level elements and lower-level elements in a hierarchy within the problem model is not only allowed, but actively supported in ANP based models. It is reflected, that not only does the importance of the criteria determine the evaluation of the alternatives as in a hierarchy, but also the evaluation of the alternatives determines the importance of the criteria of the model. Priorities are computed using pairwise comparisons and judgment in the same way as in the case of AHP. The feedback structure looks more like a network, with cycles connecting its components of elements, and with loops that connect a component to itself. The network also has sources and sinks. A source node is an origin of paths of influence in the network and never a destination of such paths, while a sink node is a destination of paths of influence and never an origin of such paths.

Decision problems involving feedback arise often in practice. Models with feedback can show us what we have to do now to attain a desired future. The challenge is to determine the priorities of the elements in the network and in particular the alternatives of the decision and even more to justify the validity of the outcome (Saaty, 2005).

\subsection{Cognitive Hierarchy Process}

The basic idea of the Cognitive Hierarchy Process (CHP) is similar to the AHP method, but CHP uses individual evaluations for sets of criteria in every alternative (Gavalec \& Mls, 2006). In comparison with the AHP method, CHP uses a similar approach and preserves the advantages of AHP, while adding some useful features. CHP uses cognitive maps for better analysis of preferences of the human decision-maker, thus helping him/her to specify and quantify the relative importance values more accurately (the network 
structure instead of the tree structure of the model is allowed). Moreover, in the case when there are many different decision-makers, e.g. customers, managers..., CHP enables using an individual cognitive map for each of them. This feature of CHP helps even the inexperienced users to express their individual preferences adequately. Fuzzy sets in CHP are useful for processing linguistic variables or other soft data in the decision process.

\subsubsection{Cognitive Map}

Cognitive map (CM) as a modeling tool was introduced by Axelrod as a system consisting of the set of concepts and the set of causal relationships (Axelrod, 1976). Each particular concept influences other related concepts via causal relationships in positive or negative sense, and there are no interactions between independent concepts. A cognitive map can be represented by a directed graph, where concepts of the CM correspond to nodes of the graph and causal relationships correspond to arcs oriented from the cause concepts to the effect concepts. Causality strength is expressed by signs + and - as positive or negative dependence between concepts.

\subsubsection{Fuzzy cognitive map}

The concept of Fuzzy cognitive map (FCM) was introduced by Kosko (Kosko, 1986) as a generalization of Axelrod's cognitive map. Fuzzy logic helps to solve problems with respect to non-precise humans' way of thinking. Simple FCM works on the principle that the causal relationships and concepts are accompanied by a number within the real unit interval $<0,1>$. By this evaluation, fine dependencies in causal relationships can be expressed and partial activation of concepts can be used, in contrary to the binary activation in CM. Theoretical basis of FCMs has been subsequently elaborated by many authors, (Kim \& Lee, 1998), (Park \& Kim, 1995), (Tsadiras \& Margaritis, 1997), for example.

In this paper, a FCM is formally defined as an ordered pair $\boldsymbol{M}=(\boldsymbol{C}, \boldsymbol{A})$, where $\boldsymbol{C}$ is a finite set of cardinality $|\boldsymbol{C}|=n$, whose elements are called concepts, and $\boldsymbol{A}$ is a matrix of type $n \times n$ with values in the real interval $<0,1>$ (alternatively, in $<-1,1>$ ). Elements of matrix $\boldsymbol{A}$ are interpreted as the levels of causal relations between pairs of concepts in $\boldsymbol{C}$. Further, we shall consider an evaluation vector of the fuzzy cognitive map $\boldsymbol{M}$, defined as a mapping $e: \boldsymbol{C} \rightarrow<0,1>$ and its values are interpreted as activation levels of concepts in $\boldsymbol{C}$. Decision support, and model behavior prediction as well, represent the most often cited domains of FCMs utilization, see (Khan, Chong \& Gedeon, 2000), (Liu, \& Satur, 1999), (Salmeron, 2009). FCMs as a supporting tool for decision making process were considered in (Gavalec, \& Mls, 2003).

\subsubsection{Decision support by AHP and CHP}

A standard approach in decision making is based on dividing the decision problem into smaller parts (alternatives, criteria, goals). Evaluations of the importance degree of various objectives and preferences for alternative solutions are then used to find the final decision. While the well-known AHP methodology uses a relative normalization approach, in which the total sum of the weights of all sub-criteria for a given parent criterion is equal to 1 , see (Golden, Harker, \& Wasil, 1989), the cognitive hierarchy process (CHP), described in (Gavalec, \& Mls, 2006), utilizes evaluation of complex systems of objectives described by fuzzy cognitive maps. In contrast to the AHP method, which works with fixed evaluation of relative weights of different alternatives (cases), CHP uses a specific system of weights for every individual alternative. CHP works with a system of individual FCM's unified by a common template.

We say that a fuzzy cognitive map $\boldsymbol{M}^{*}=\left(\boldsymbol{C}^{*}, \boldsymbol{A}^{*}\right)$ is a template for a system $\boldsymbol{M}=\left(\boldsymbol{M}_{s} ; s \in \boldsymbol{S}\right)$ of individual FCM's:

$\boldsymbol{M}_{s}=\left(\boldsymbol{C}_{s}, \boldsymbol{A}_{s}\right)$, if $\boldsymbol{C}_{s}=\boldsymbol{C}^{*}$ and $\boldsymbol{A}_{s} \leq \boldsymbol{A}^{*}$ holds true for every individual $s \in \boldsymbol{S}$. 
For the purpose of CHP, a tree structure of the template $\boldsymbol{M}^{*}$, similarly to AHP, is usually assumed. $C^{*}$ denotes the set of template nodes, $A^{*}$ is the set of weighted template edges and $\boldsymbol{M}^{*}$ is a root-tree with the root $c_{0} \in C^{*}$. Any node $c \in C^{*}, c \neq c_{0}$ has the unique predecessor denoted by $p(c)$, and for any node $c \in \boldsymbol{C}^{*}, \boldsymbol{S}(c)$ denotes the set of all successors of $c$. For every fuzzy cognitive map $\boldsymbol{M}_{s} \in \boldsymbol{M}$, an individual evaluation mapping $e_{s}: \boldsymbol{C} \rightarrow<0,1>$ is defined recursively, according to the tree structure of template $\boldsymbol{M}^{*}$.

\section{On-line decision making}

Decision making in real world situations are usually not a single shot process. Solving complex problems by decomposition to smaller and simpler sub-problems or making successive decisions in chains of situations can be mentioned within the context of multiple decision making. Another specific case is represented by decision making in situations, when complete set of alternatives is not available at a time, but rather in form of a flow of information revealing new opportunities and withdrawing some of the known ones. Decision maker is obliged to solve two interrelated problems in this situation - he is looking for the best alternative from the current offer, and at the same time he has to decide if to take the best alternative available at the moment, or to wait for next offers and run the risk, that the former best alternative will be lost and all coming alternatives will be worse (Gavalec, \& Mls, 2008).

\section{1 "Select a house" example}

As an example let us consider the following scenario: Moving to another city, "typical family" is looking for a new home. The new house is assumed to have several rooms, and its price should be adequate to actual technical state and expected other costs. The quality of environment is important as well. General multi-criteria model depicting the decision problem in detail is on Figure 2.

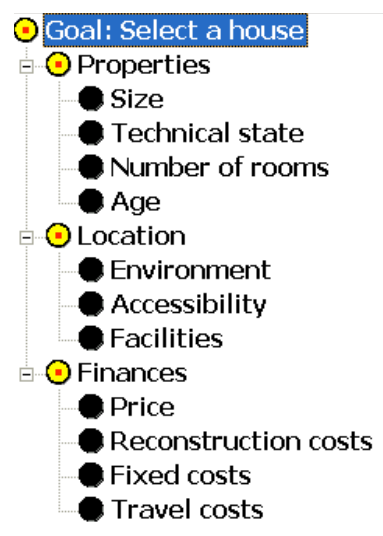

Figure 2 Multi-criteria model of the "Select a house" decision problem

In certain time, limited number of real-estate offers is available. From the point of view of our model family members, AHP method may be applied to evaluate individual offers/alternatives and to find the most suitable one. The individual preferences of family members may differ, however, so the group decision making support should be considered.

As stated in chapter 2.2, AHP model has its limitation when there is a non-trivial dependence among elements of the model. In the "Select a house" example, some of such relations are illustrated on Figure 3 by dotted lines. The new structure cannot be simply processed by standard AHP approach, ANP or CHP should be utilized. 


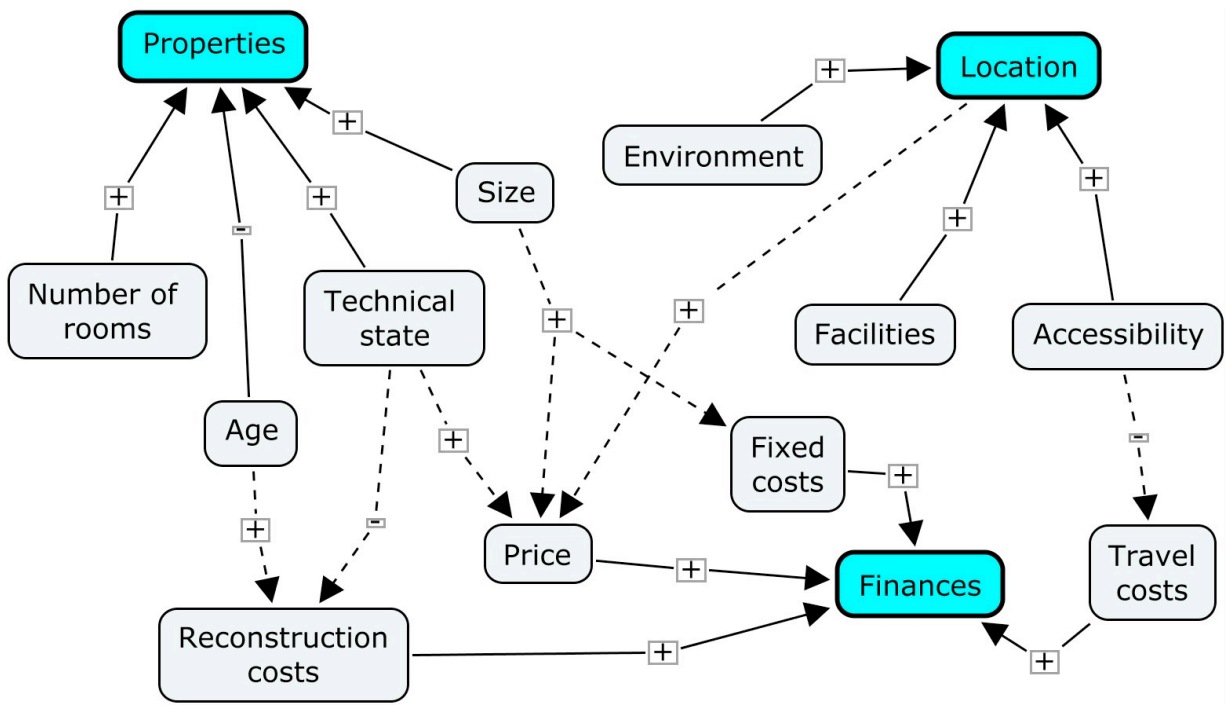

Figure 3 Networked model of the "Select a house" decision problem

In the "Select a house" example of on-line decision making, two possible approaches to final decision can be considered. First, the critical value approach based on the defined minimal acceptable evaluation of the alternative(s) in demand helps to decide, whether to accept the best rated alternative from available set of offers, or to wait for, possible more proper, future offers. The approach holds a risk that all future offers will be worse than that of present ones, so some other information is needed. This additional information is included in historical data, in time series of past alternatives evaluation. So, the second step is to compute the trend and eventual periodical components of the past data set, that may help to better understand the real-estate market and consequently to adopt better decision. In the aforesaid context, predictive potentiality of the ANP and CHP stands for an advantage in on-line decision situations.

\section{Conclusions and future work}

Several approaches to decision support were discussed in the paper. Analytic hierarchy process were taken as a standard method and it was compared with Analytic network process and Cognitive hierarchy process on the "Select a house" decision problem. Tree structure of the AHP model was confronted with the network version of the CHP model of the same problem. From the first experience CHP and ANP approaches seem to share some characteristics, which are advisable to be examined in more detail. Specific conditions of on-line decision making, when incomplete set of alternatives is available in a given time period, together with multiple decision makers approach were mentioned to point out the complexity of real problems.

Further improvement of the decision process, introduced in the paper can be attained, if an expert knowledge of real-estate broker is availed of. It poses a problem of processing relevant data, which will be sooner or later unmanageable for human expert. An autonomous agent, fitted with decision makers' priorities, is expected to manage with both information overload, and variations and uncertainty of humans' request. 


\section{REFERENCES}

Alter, S.L. (1975). A Study of Computer Aided Decision Making in Organizations. Ph.D. dissertation, M.I.T.

Axelrod, R. (1976). Structure of Decision: The Cognitive Maps of Political Elites. Princeton University Press, New Jersey.

Bekey, G. A. (2005). Autonomous Robots: From Biological Inspiration to Implementation and Control (Intelligent Robotics and Autonomous Agents). The MIT Press.

David, F. (1989). Strategic Management. Columbus: Merrill Publishing Company.

Drucker, P. F., Hammond, J., Keeney, R., Raiffa, H. \& Hayashi, A. M. (2001). Harvard Business Review on Decision Making. Harvard Business School Press.

Fernández-Aráoz, C. (2007). Great People Decisions: Why They Matter So Much, Why They are So Hard, and How You Can Master Them. Wiley.

Gavalec, M., \& Mls, K. (2003). Fuzzy cognitive maps and decision making support. Proc. of the 21st Conf. Mathem. Methods in Economics, Czech University of Agriculture in Prague, 87-93.

Gavalec, M., \& Mls, K. (2006). Cognitive hierarchy process - an approach to decision making support. Proc. of the 24th Conf. Math. Methods in Economics, 187-193.

Gavalec, M., \& Mls, K. (2008). On-line fuzzy optimization based on cognitive hierarchy process. Abstracts of the 9th Inter. Conf. Fuzzy Sets Theory and its Applications, Liptovský Ján, 46-46.

Golden, B.L., Harker, P.T., \& Wasil, E.A. (1989). The analytical hierarchy process - applications and studies. Springer Verlag, New York.

Holland, J. M. (2003). Designing Autonomous Mobile Robots: Inside the Mind of an Intelligent Machine. Newnes.

Hussey, D. (2001). Creative Strategic Thinking and the Analytical Process: Critical Factors for Strategic Success, Strategic Change, 10/4:201-213.

Jedermann, R., Antúnez Congil, L. J., Lorenz, M., Gehrke, J. D., Lang, W. \& Herzog, O. (2007). Dynamic Decision Making on Embedded Platforms in Transport Logistics - A Case Study. International Conference on Dynamics in Logistics, H.-D. Haasis, H.-J. Kreowski and B. Scholz-Reiter, Eds., Bremen, Germany, Aug. 28-30, pp. 191-198.

Keller, P. W., Duguay, F.-O. \& Precup, D. (2004). RedAgent-2003: An Autonomous Market-Based Supply-Chain Management Agent. Third International Joint Conference on Autonomous Agents and Multiagent Systems - Volume 3 (AAMAS'04), pp. 1182-1189.

Khan, M.S., Chong, A., \& Gedeon, T. (2000). A Methodology for Developing Adaptive Fuzzy Cognitive Maps for Decision Support. Journal of Advanced Computational Intelligence, 4(6)

Kim, H., \& Lee, K. (1998). Fuzzy implications of fuzzy cognitive map with emphasis on fuzzy causal relationship and fuzzy partially causal relationship. Fuzzy Sets and Systems. 97(3): 303-313. 
Kosko, B. (1986). Fuzzy cognitive maps. International Journal of Man-Machine Studies, 24(1): 65-75.

Liu, Z., \& Satur, R. (1999). Contextual fuzzy cognitive map for decision support in geographic information systems. IEEE Transactions on Fuzzy Systems. 7(5): 495-507

Mintzberg, H., \& Quinn, J. B. (1988). The Strategy Process. Prentice-Hall, Harlow.

Mullins, J., Walker, O. C. Jr., \& Boyd, H. W. (2006). Marketing Management: A Strategic DecisionMaking Approach. McGraw-Hill/Irwin.

Park, K., \& Kim, S. (1995). Fuzzy Cognitive Maps Considering Time Relationships. International Journal of Human-computer Studies. 42(2): 157-168.

Pollock, J. L. (2006). Against Optimality: Logical Foundations For Decision-Theoretic Planning In Autonomous Agents. Computational Intelligence, 22/1:1-25.

Power, D. J. (2002) Decision Support Systems: Concepts and Resources for Managers. Westport, CT, Greenwood/Quorum.

Saaty, T.L. (1980). The analytic hierarchy process. McGraw-Hill, New York.

Saaty, T.L. (2005). Theory and Applications of the Analytic Network Process: Decision Making with Benefits, Opportunities, Costs, and Risks. RWS Publications.

Salmeron, J.L. \& Herrero, I. (2005). An AHP-based methodology to rank Critical Success Factors of Executive Information Systems. Computer Standards and Interfaces 28(1), pp.1-12.

Salmeron, J.L. (2009). Supporting decision makers with Fuzzy Cognitive Maps. Research-Technology Management 52(3), pp. 53-59.

Scott Morton, M.S. (1967). Computer-Driven Visual Display Devices. Their Impact on the Management Decision-Making Process, Doctoral Dissertation, Harvard Business School.

Sprague, R. H., Jr., \& Carlson, E. D. (1982). Building Effective Decision Support Systems. Englewood Cliffs, N.J.: Prentice-Hall, Inc.

Torra, V., Narukawa, Y., \& Yoshida, Y. eds. (2007). Modeling Decisions for Artificial Intelligence. Lecture Notes in Computer Science. Springer.

Thrun, S. (2005). Probabilistic Robotics (Intelligent Robotics and Autonomous Agents). The MIT Press.

Tsadiras, A.K., \& Margaritis, K.G. (1997). Cognitive Mapping and Certainty Neuron Fuzzy Cognitive Maps. Information Sciences (1997) 101: p. 109-130.

Turban, E. (1995). Decision support and expert systems: management support systems. Englewood Cliffs, N.J., Prentice Hall. 